\title{
A novel multimessenger study of Starburst galaxies: implications for neutrino astronomy
}

\section{Antonio Marinelli, ${ }^{a, *}$ Antonio Ambrosone, ${ }^{a, b}$ Marco Chianese, ${ }^{a, b}$ Damiano Fiorillo, ${ }^{a, b}$ Gennaro Miele $^{a, b, c}$ and Ofelia Pisanti ${ }^{a, b}$}

${ }^{a}$ INFN - Sezione di Napoli,

Complesso Univ. Monte S. Angelo, I-80126 Napoli, Italy

${ }^{b}$ Dipartimento di Fisica "Ettore Pancini" , Universitá degli studi di Napoli "Federico II",

Complesso Univ. Monte S. Angelo, I-80126, Napoli, Italy

${ }^{c}$ Scuola Superiore Meridionale, Universitá degli studi di Napoli “Federico II”,

Largo San Marcellino 10, 80138 Napoli, Italy

E-mail: antonio.marinelli@na.infn.it

Starburst galaxies (SBGs) and more in general starforming galaxies (SFGs) represent a class of galaxies with a high star formation rate (up to $100 \mathrm{Mo} /$ year). Despite their low luminosity, they can be considered as guaranteed "factories" of high energy neutrinos, being "reservoirs" of accelerated cosmic rays and hosting a high density target gas in the central region. The estimation of their point-like and diffuse contributions to the neutrino astrophysical flux measured by IceCube can be crucial to describe the diffuse neutrino spectral features as well as the peculiar point-like excess like NGC1068. To this aim we use the latest gamma-ray catalog of this class of objects to perform a multimessenger study and describe their gamma-ray emission through a calorimetric scenario. For the diffuse analysis we perform a blending of the measured spectral indexes and obtain a multi-component description of extragalactic background light (EGB), high energy starting events (HESE) and high-energy cascade IceCube data. Remarkably, we find that, differently from recent prototype scenarios, the spectral index blending allows starburst galaxies to account for up to $40 \%$ of the HESE events at $95.4 \% \mathrm{CL}$ and favors a maximal energy of the accelerated cosmic rays at tens of PeV. For the point-like analysis we apply the calorimetric approach to the known SBGs within $100 \mathrm{Mpc}$, considering, where possible, a source-by-source description of the star formation rate. These results are then compared with what can be expected from IceCube, IceCube/Gen2 and the incoming $\mathrm{KM} 3 \mathrm{NeT}$.

$37^{\text {th }}$ International Cosmic Ray Conference (ICRC 2021)

July 12th-23rd, 2021

Online - Berlin, Germany

\footnotetext{
${ }^{*}$ Presenter
} 


\section{Introduction}

Despite the existing limits, starburst galaxies (SBGs) are well-motivated candidates for the diffuse neutrino flux, since they are guaranteed high-energy cosmic-ray "reservoirs" with enough interstellar gas to be considered as good calorimeters. Observed and catalogued mostly through their infrared emission, denoting an intense star formation rate, gamma-ray observations for few of them point out their capability to emit non-thermal component above the TeV energy. The possibility of confining cosmic-rays in a core with the high-density interstellar matter guarantees an effective hadronic contribution to the observed gamma-ray emission. However, the gammaray observations from these non-wind galaxies are available just from few of them, confirming their low luminosity at high energies and making difficult an exhaustive prediction of the neutrino counterpart. Recent works [19, 22] have reexamined the neutrino and gamma-ray emissions from SBGs pointing out that these sources can indeed account for the IceCube through-going muon neutrino flux at hundreds of $\mathrm{TeVs}$ in agreement with gamma-ray data. In particular, they have proposed a prototype-based method to compute the cumulative neutrino and gamma-ray fluxes emitted from the SBGs population. In this approach, the galaxy M82 is considered as a reference SBG for setting the physical parameters such as supernovae rate, magnetic field, velocity of the wind, density of the interstellar medium. Most importantly, the cosmic-ray spectrum injected in all the SBGs is fixed to be a power-law with spectral index of $4.2^{1}$ and cut-off energy of $100 \mathrm{PeV}$. Although this scenario provides a good description of the through-going muon neutrino flux, the astrophysical neutrino flux below $100 \mathrm{TeV}$ remains still unexplained. Moreover, at higher neutrino energies, a contribution from blazars to the diffuse neutrino flux is generally expected. In this ICRC contribution we go through two different multi-messenger and multi-component analyses of SBGs (diffuse and point-like) to obtain the expected neutrino emission.

For the diffuse emission analysis we relax the assumption of a single power-law and consider a more realistic scenario where the cosmic-ray spectra of each starburst galaxy can have different spectral indexes. In particular, we go through the recent study [8] that considers ten years of Fermi-LAT data for a sample of 12 starforming galaxies making a statistical analysis with the spectral features of these astrophysical objects. Differently from [22], we take into account the distribution of spectral indexes from this sample and consider it as representative of the whole SBGs population. Such a data-driven blending of spectral indexes has the remarkable results of increasing the neutrino flux at $100 \mathrm{TeV}$ without enlarging the gamma-ray flux below $1 \mathrm{TeV}$. This is the right behaviour required to potentially explain IceCube low-energy events and alleviate the tension between neutrino and gamma-ray data. To further investigate this result, we perform a multi-messenger likelihood analysis of the extragalactic gamma-ray background (EBL) measured by Fermi-LAT and the neutrino flux observed by IceCube, considering both the contributions of SBGs and blazars. For the latter, we follow the model [20] that, using the TXS0506+056 as a standard candle, describes the neutrino and electromagnetic emission satisfying the IceCube stacking limit [2]. For both the two classes of sources, we include the secondary gamma-ray emission from electromagnetic cascades using the public code $\gamma$-Cascade. Regarding neutrino data, we examine the latest 7.5-year HESE data as well as the 6-year high-energy cascade ones which probe the neutrino emission at lower neutrino

\footnotetext{
${ }^{1}$ It is important to remark that, in general, when we are referring to the spectral index, we usually refer to the index of high-energy protons.
} 
energies. In order to analyze the whole EGB spectrum, we also take into account the diffuse gamma-ray emission from radio galaxies dominating the Fermi-LAT observations below $1 \mathrm{GeV}$ [9]. Along with the three overall normalizations for the SBGs, blazars and radio galaxies components, we leave as a free parameter the maximal energy reached by the cosmic rays accelerated from supernovae remnants (SNRs) inside of the nucleus of starburst galaxies. In this analysis, we focus on highlighting the differences in the results obtained with the two different models for the SBG emission: the standard prototype approach resulting in a single power-law behavior and the more realistic data-driven blending of spectral indexes. We find that, independently of the data-sets considered in the multi-messenger analysis, the former is more constrained by data and implies an almost negligible SBG contribution to the diffuse neutrino flux. On the other hand, the latter is in better agreement with data and a sizeable SBG neutrino component is allowed at $100 \mathrm{TeV}$. Moreover, the SBG model with blending generally requires a cut-off energy smaller than few tens of $\mathrm{PeV}$, in agreement with an expected dominant neutrino emission from blazars above $200 \mathrm{TeV}$ [20]. Finally, we point out that our model for the SBG component is allowed to account for the $40 \%$ (50\%) of the total 7.5-year HESE neutrino events at 95.4\% (99.7\%) confidence level, while being compatible with the existing limits on the non-blazar EGB component and on the contribution of nearby SBGs to the diffuse neutrino flux [16].

However, in order to explain a sizeable portion of measured high-energy neutrino flux with SFGs and SBGs, the deep Universe must be considered up to redshift $\sim 4-5$, because of their dimness [11,22]. The low gamma-ray luminosity of SFGs and SBGs typically represents a bound for their contribution to the observed astrophysical neutrinos as a point-like component. Currently, only a dozen of these sources have been catalogued as gamma-ray point-like sources using the Fermi-LAT data and only few of them have been observed through Imaging Cherenkov telescopes.

For the point-like emission analysis we employ a multi-messenger and multi-wavelength approach to assess the ability of current and upcoming neutrino telescopes to observe such galaxies as neutrino point-like sources. We follow the scenario proposed by [21] which considers the transport of high-energy protons and electrons and we analyze the 10-year Fermi-LAT data provided by [8], taking into account the spectral energy distributions (SEDs) of 13 SFGs and SBGs. We describe these gamma-ray observations through the hadronic and leptonic processes at work in the starforming regions and determine the corresponding neutrino fluxes. For each SBG, we require the star formation rate (SFR) to be consistent with the one derived from infra-red (IR) and ultra-violet (UV) observations [13] within a maximal level of discrepancy, thus making our predictions more robust. Hence, we compare the most-likely neutrino flux normalizations at $1 \mathrm{TeV}$ with IceCube, IceCube-Gen2, and KM3NeT sensitivities (see Figure 2).

\section{Emissions of a single Starburst galaxy}

SBGs are characterized by a high star formation rate (SFR) $\left(\psi \sim 10-100 \mathrm{M}_{\odot} \mathrm{yr}^{-1}\right)$ which highlights the abundance of cosmic-ray accelerators as well as a higher density of interstellar gas which represents the target for inelastic collision of accelerated particles. Since interstellar gas efficiently absorbs star emission and re-emits it in the infrared (IR), the IR emission of SBGs, $10-100$ times greater than normal galaxies, can be considered a good tracer for the SFR. These characteristics favour the production of high-energy gamma-rays and neutrinos through the hadronic 
proton-proton interaction and explain the linear relation between gamma-ray luminosity and infrared emission observed by [23]. Beside an interstellar medium density of $n_{\text {ISM }} \sim 10^{2} \mathrm{~cm}^{-3}$, these sources present also a strong magnetic field $\left(10^{2}-10^{3} \mu \mathrm{G}\right)$ [24] which plays an important role in the CR confinement. The episodes of starburst are caused by supernova explosions and usually happen in a region called starburst nucleus (SBN). In particular, SNRs are expected to inject a massive amount of gas and this becomes a supersonic wind flow [12]. Hence, winds and turbulence play a decisive role in the motion and interaction of CRs. If these high-energy CRs are confined inside these galaxies, SBGs could be thick enough to efficiently produce neutrinos and non-thermal radiation. This calorimetric condition can be expressed by

$$
T_{\mathrm{loss}} \leq T_{\mathrm{esc}},
$$

where $T_{\text {loss }}$ is the typical CR timescale for interactions and $T_{\text {esc }}$ is the timescale taken for a CR to escape the source. Many authors focused on CR spectral features of SBGs [18] inferring that CR electrons are well confined inside SBNs, while the calorimetric condition for high-energy protons strictly depends on the ISM density and the wind flow velocity inside a SBG (see also [22]). In this paper, we follow [21] to describe CR timescales. We consider the SBNs as a spherical region with the advection time $T_{\mathrm{adv}}=R / v_{\text {wind }}$ depending on the radius $(R)$ of this region and on the wind velocity $\left(v_{\text {wind }}\right)$. On the other hand, for the time loss of CR we take into account the protonproton $(p-p)$ interaction. We describe CR diffusion through a Kolmogorov-like scenario, assuming a density of the magnetic field $F(k) \propto k^{-d+1}$ with $d=5 / 3$ and a regime of strong turbulence inside the SBN [22]. These assumptions lead to a diffusion coefficient $D(p) \propto p^{1 / 3}$, which implies $T_{\text {diff }}(E) \propto E^{-1 / 3}$. For a magnetized fluid the Kolmogorov scenario should in principle be replaced by the Kraichnan model [14] for turbulence. However, as shown by [21, 22], the diffusion timescale is always larger than all the other timescales, so that the details of the turbulence model do not influence our conclusions. The escape time $T_{\text {esc }}$ in Eq. (1) is given by

$$
T_{\mathrm{esc}}=\left(\frac{1}{T_{\mathrm{adv}}}+\frac{1}{T_{\mathrm{diff}}}\right)^{-1} .
$$

For SBG typical values $\left(R \sim 10^{2} \mathrm{pc}\right.$ and $v_{\text {winds }} \sim 10^{2}-10^{3} \mathrm{~km} / \mathrm{s}$, see [24]), we have that $T_{\text {adv }} \sim 10^{5}-10^{6} \mathrm{yr}$. On the other hand, the high level of turbulence and interstellar medium density makes the diffusion timescale much greater than this timescale (see [21]); consequently, winds are principally dominated by advection and therefore $T_{\text {esc }} \simeq T_{\text {adv }}$. The timescale $T_{\text {loss }}$ depends on the proton energy and in particular, for energies much greater than the proton mass, it is mainly driven by the timescale of $p$ - $p$ interactions. As shown in [21], $T_{\text {loss }}$ usually becomes less than advection timescale for energy higher than $10 \mathrm{TeV}$. On the other hand, electrons are always confined inside the SBN and therefore, they always lose efficiently their energy. The most convenient way to study the CR distribution (high-energy protons and primary electrons) inside SBNs is to use the leaky-box model equation

$$
F_{p, e}=Q_{p, e}\left(\frac{1}{T_{\mathrm{adv}}}+\frac{1}{T_{\mathrm{loss}}}+\frac{1}{T_{\mathrm{diff}}}\right)^{-1}
$$

where $F_{p, e}$ and $Q_{p, e}$ are respectively the distribution function and the injection rate of protons and electrons. Eq. (3) physically represents the balance between the injection and CRs loss terms. 
Indeed, the high ISM density, from one hand, fuels the star forming activity and at the same time, it is heated by supernovae explosions. Hence, a balance between the injection and the winds phenomena is expected in a generic SBN. The injection of CRs arises directly from SNRs, consequently $Q_{p, e}\left(p, \mathcal{R}_{\mathrm{SN}}, \alpha, p^{\max }\right)$ depends both on the rate of supernova explosions $\mathcal{R}_{\mathrm{SN}}$ and on the spectral shape given by a single SNR.

It is worth noticing that the calorimetric condition given by Eq. (1) significantly affects the spectrum of expected neutrinos and gamma-rays [22]. In fact, we have that $Q_{v, \gamma} \propto Q_{\pi} \propto n_{\mathrm{ISM}} \sigma_{p p} F_{p}$ and $F_{p}$ principally depends on the minimum timescale in Eq. (3). In particular, we get

$$
Q_{v, \gamma} \propto \begin{cases}Q_{p} & \text { Calorimetric Scenario } \\ c n_{\mathrm{ISM}} \sigma_{p p} Q_{p} \frac{R}{v_{\mathrm{wind}}} & \text { Wind Scenario }\end{cases}
$$

This equation highlights that in the calorimetric scenario the gamma-ray and neutrino emission from a SBG is weakly dependent on the physical parameters of the source. Indeed, in this scenario we are able to quantify the hadronic emission with three main quantities: $\mathcal{R}_{\mathrm{SN}}, \alpha$ and $p^{\max }$. Keeping in mind the direct relation between $\mathcal{R}_{\mathrm{SN}}$ and the star formation rate (SFR) $\psi$ ([22]), for the rest of the paper we continue to describe the emission of the SBGs through: $\psi, \alpha$ and $p^{\max }$. This is a crucial observation, because the calorimetric approximation allows us to neglect all the structural details of the sources and consequently, just like in Ref. [22], it is possible to fix all the other parameters such as the magnetic field, the velocity of the wind, the density of the interstellar medium, to the values of a benchmark galaxy, which is M82. A crucial role in this study is played also by the the maximum proton energy expected in SBNs, which is here considered as free parameter in the following multi-messenger likelihood analysis. Several authors discuss this point however the possibility for the SNRs and fresh star formation regions to accelerate CRs up to the knees energy is still an open question.

\section{Diffuse analysis}

In order to quantitatively discuss the role of starburst galaxies in the production of astrophysical neutrinos, we perform a statistical multi-messenger analysis which takes into account both neutrino and gamma-ray data. In particular, we analyze two neutrino IceCube data samples: the 7.5-year HESE data and the 6-year high-energy cascade data. The former contains neutrino events of all flavours with track and shower topologies above $60 \mathrm{TeV}$. The latter, instead, only includes showerlike events (mostly electron and tau neutrino flavours) and characterizes the diffuse neutrino flux down to few $\mathrm{TeV}$ thanks to the smaller background contamination. Concerning gamma-ray data, we examine the extragalactic gamma-ray background (EGB) measured by Fermi-LAT [6]. Most of the EGB spectrum is accounted by for resolved and unresolved blazars, while the contribution of other sources is in general sub-dominant [15]. Therefore, in addition to the starburst galaxies, we take into account other classes of sources contributing to the neutrino and gamma-ray skies, in details for the gamma rays we consider additional components from blazars and radio galaxies while for neutrino we consider only a additional component from blazars.

For both the neutrino and the gamma-ray components, we perform a maximum likelihood analysis 

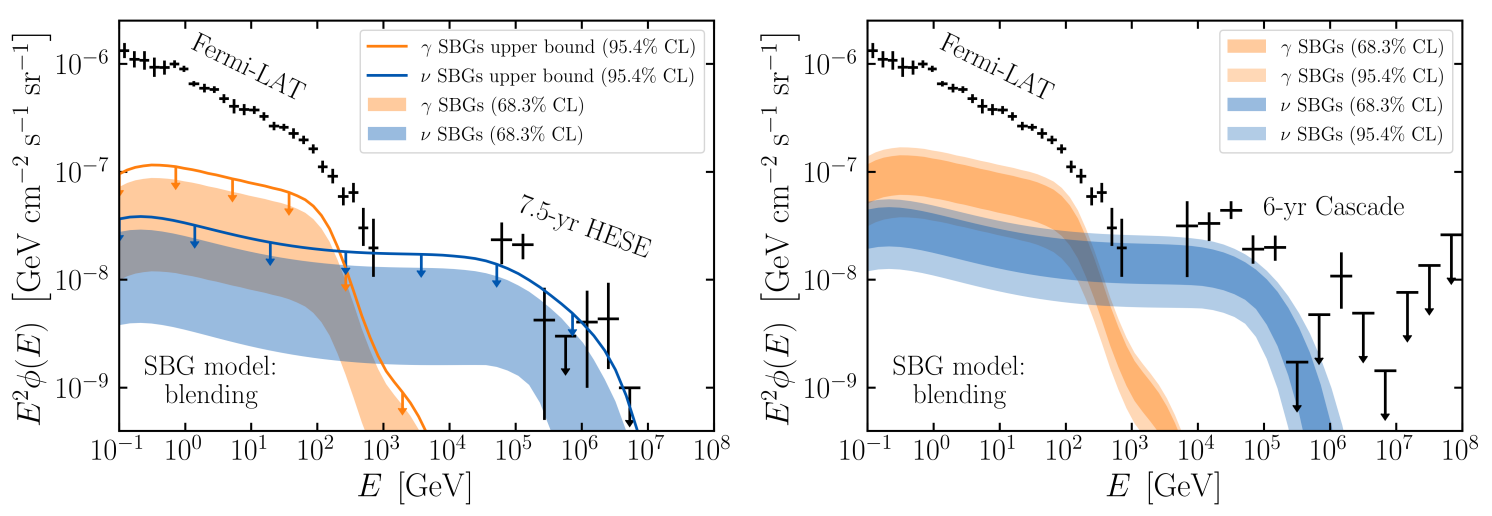

Figure 1: Gamma-ray (orange) and single-flavour neutrino (blue) uncertainty bands at 68.3\% CL (dark colors) and $95.4 \%$ CL (light colors) for the SBG component deduced by the multi-messenger analysis in case of data-driven blending of spectral indexes. The left (right) plot corresponds to the multi-messenger analysis with IceCube 7.5-year HESE (6-year cascade) neutrino data. In the left plot, the solid lines correspond to upper bounds at $95.4 \% \mathrm{CL}$.

using a chi-squared likelihood. For the neutrino data, we use the following chi-squared function:

$$
\chi_{v}^{2}=\sum_{i}\left(\frac{\Phi_{v, i}^{\mathrm{IC}}-N_{\mathrm{Blazars}} \Phi_{v, i}^{\mathrm{Blazars}}-N_{\mathrm{SBG}} \Phi_{v, i}^{\mathrm{SBG}}\left(p^{\mathrm{max}}\right)}{\sigma_{v, i}^{\mathrm{IC}}}\right)^{2},
$$

where $\Phi_{v, i}^{\mathrm{IC}}$ is the diffuse single-flavour flux observed by IceCube in each energy interval $i$ with uncertainties $\sigma_{v, i}^{\mathrm{IC}}$, whereas $\Phi_{v, i}^{\mathrm{Blazars}}$ and $\Phi_{v, i}^{\mathrm{SBG}}$ are the neutrino flux of blazars and SBG sources, respectively. The resulted neutrino spectra considering EGB, HESE and cascade data are reported in Fig. 1.

\section{Point-like analysis}

Our main purpose is to determine the high-energy neutrino emission from SFGs and SBGs based on a likelihood analysis of gamma-ray data. A crucial observation must be made: for some of these galaxies, a possible additional source of gamma-rays may be related to AGN activity [17], which is not included in the following analysis. Thus we test the hypothesis that the observed gammaray SEDs are saturated by star-forming activity only, and determine the most-likely neutrino emission under this assumption. We analyze the gamma-ray SEDs of 13 galaxies observed by Fermi-LAT with 10 years of observation [8]. ${ }^{2}$ For M82 and NGC 253 we make also use of the data provided by VERITAS [5] and H.E.S.S. [4], respectively. For each galaxy, we pursue a Bayesian approach to assess the most-likely values for the two free parameters of the model: the star formation rate $\dot{M}_{*}$ and the spectral index $\Gamma$ of injected protons and electrons. We determine the posterior distribution as

$$
p\left(\dot{M}_{*}, \Gamma \mid \mathrm{SED}\right) \propto p\left(\mathrm{SED} \mid \dot{M}_{*}, \Gamma\right) p\left(\dot{M}_{*}\right) p(\Gamma),
$$

\footnotetext{
${ }^{2}$ The data reported in [8] are normalized to a constant value of $10^{-9} \mathrm{GeV} \mathrm{cm}^{-2} \mathrm{~s}^{-1}$ at an energy of $1 \mathrm{GeV}$. We have suitably rescaled them using the reported best-fit parameters of the power-law model.
} 


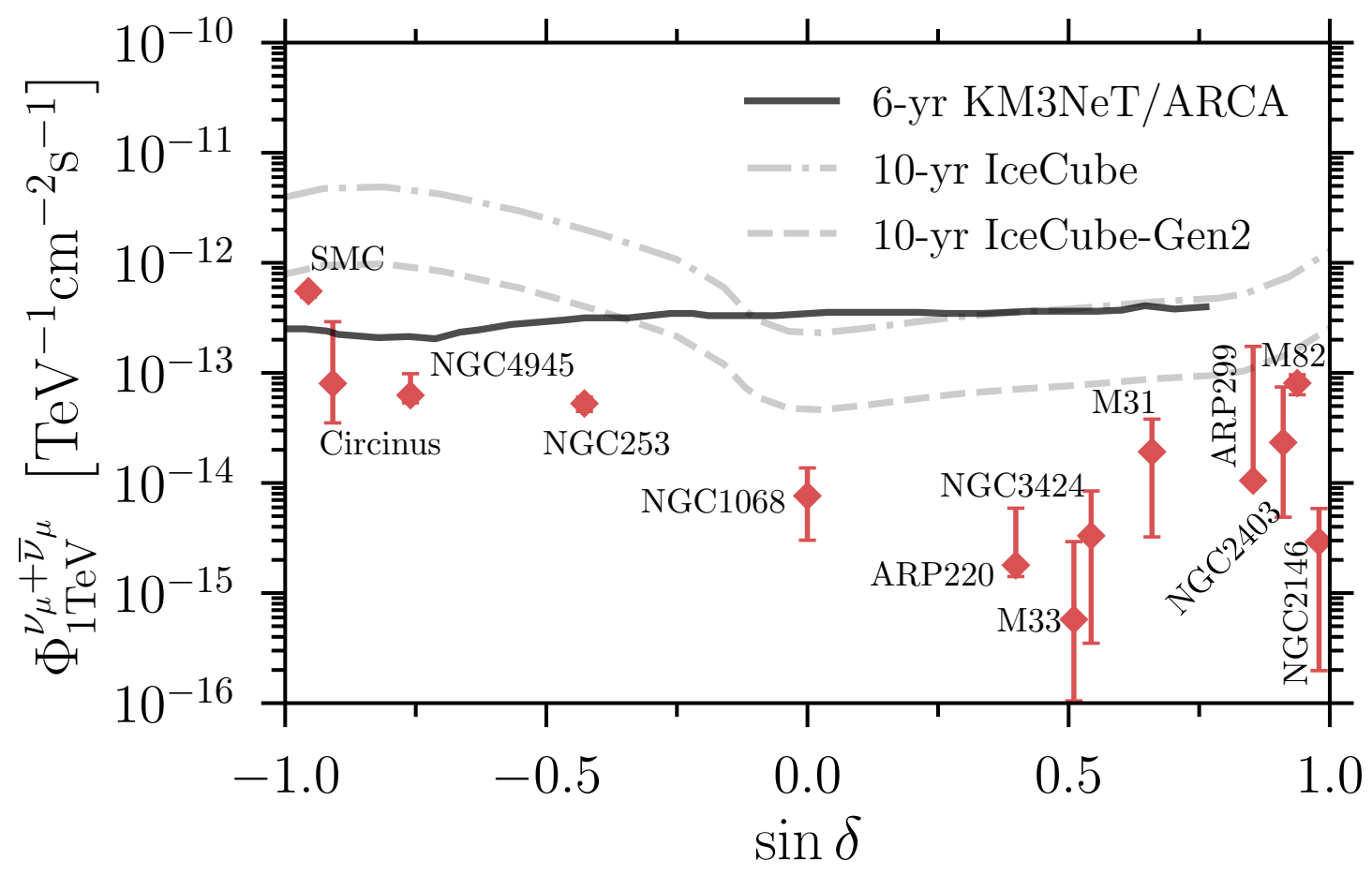

Figure 2: Muon neutrino flux normalizations at $1 \mathrm{TeV}$ predicted by the source star-forming activity as a function of source declination. The diamonds correspond to the most-likely values of the source parameters deduced by current gamma-ray data [10], while the bands represent the $68 \%$ credible intervals of the marginal flux distributions. The lines shown the point-like sensitivity of different neutrino telescopes: 6-year KM3NeT/ARCA [7], 10-year IceCube [3], and 10-year IceCube-Gen2 estimated according to [1].

with a Gaussian likelihood function

$$
p\left(\operatorname{SED} \mid \dot{M}_{*}, \Gamma\right)=e^{-\frac{1}{2} \sum_{i}\left(\frac{\operatorname{sED}_{i}-E_{i}^{2} \Phi_{\gamma}\left(E_{i} \mid \dot{M}_{*}, \Gamma\right)}{\sigma_{i}}\right)^{2}} .
$$

Here, $\mathrm{SED}_{i}$ are the measured data, where $i$ runs over the energy bins centered around the energy $E_{i}$, and $\sigma_{i}$ are the observational uncertainties. The data are compared to the gamma-ray emission $\Phi_{\gamma}\left(E_{i} \mid \dot{M}_{*}, \Gamma\right)$ predicted by our model. We adopt the source distances provided by [13] and neglect the uncertainty on such values. For all the galaxies we consider the same uniform prior on the spectral index in the range 1.0-3.0. For the SFR, we instead adopt a different uniform prior distribution for each source. We require $\dot{M}_{*}$ to be consistent within a factor of 3 with the corresponding values reported in [13]. This choice is made to cover the wide variety of SFR estimates presented in the literature [8]. To compare the values of $\dot{M}_{*}$ with the one measured we follow the relation between $\dot{M}_{*}$ and the radiation density reported in [10]. The results of the analysis are summarized in Fig. 2.

\section{Results and conclusions}

Event though the nearby known SBGs can contribute at the level of $\sim \%$ to the whole sky astrophysical flux measured by IceCube, the upcoming neutrino telescopes will potentially observe 
nearby some star-forming and starburst galaxies as point-like sources. In particular, they could pose a solid link between the hadronic emission of these sources, supposed to dominate the GeV$\mathrm{TeV}$ gamma-rays, and the expected intense star-forming activity as obtained from IR and UV observations. On the other hand whenever we extend the analysis of SBG neutrino emission to the deep sky $(z \sim 4)$ the total contribution to IceCube measurements can rise up to $20 \%-40 \%$ of the measured neutrino astrophysical flux.

\section{References}

[1] M. G. Aartsen et al. IceCube-Gen2: the window to the extreme Universe. J. Phys. G, 48(6):060501, 2021.

[2] M.G. Aartsen et al. The contribution of Fermi-2LAC blazars to the diffuse TeV-PeV neutrino flux. Astrophys. J., 835(1):45, 2017.

[3] M.G. Aartsen et al. Time-Integrated Neutrino Source Searches with 10 Years of IceCube Data. Phys. Rev. Lett., 124(5):051103, 2020.

[4] H. Abdalla and others. The starburst galaxy NGC 253 revisited by H.E.S.S. and Fermi-LAT. Astroparticle Physics Journal, 617:A73, September 2018.

[5] V. A. Acciari and others. A connection between star formation activity and cosmic rays in the starburst galaxy M82. Nature, 462(7274):770-772, December 2009.

[6] M. Ackermann et al. The spectrum of isotropic diffuse gamma-ray emission between 100 $\mathrm{MeV}$ and $820 \mathrm{GeV}$. Astrophys. J., 799:86, 2015.

[7] S. Aiello et al. Sensitivity of the KM3NeT/ARCA neutrino telescope to point-like neutrino sources. Astropart. Phys., 111:100-110, 2019.

[8] M. Ajello, M. Di Mauro, V.S. Paliya, and S. Garrappa. The $\gamma$-ray Emission of Star-Forming Galaxies. Astrophys. J., 894(2):88, 2020.

[9] M. Ajello et al. The Origin of the Extragalactic Gamma-Ray Background and Implications for Dark-Matter Annihilation. Astrophys. J. Lett., 800(2):L27, 2015.

[10] Antonio Ambrosone, Marco Chianese, Damiano F. G. Fiorillo, Antonio Marinelli, and Gennaro Miele. Could nearby star-forming galaxies light up the point-like neutrino sky? arXiv e-prints, page arXiv:2106.13248, June 2021.

[11] Antonio Ambrosone, Marco Chianese, Damiano F. G. Fiorillo, Antonio Marinelli, Gennaro Miele, and Ofelia Pisanti. Starburst galaxies strike back: a multi-messenger analysis with Fermi-LAT and IceCube data. Mon. Not. Roy. Astron. Soc., 503(3):4032, 2021.

[12] R.A. Chevalier and Andrew W. Clegg. Wind from a starburst galaxy nucleus. Nature, 317:44, 1985. 
[13] P. Kornecki, L. J. Pellizza, S. del Palacio, A. L. Müller, J. F. Albacete-Colombo, and G. E. Romero. $\gamma$-ray/infrared luminosity correlation of star-forming galaxies. Astron. Astrophys., 641:A147, 2020.

[14] Robert H. Kraichnan. Inertial-Range Spectrum of Hydromagnetic Turbulence. Phys. Fluids, 8:1385-1387, 1965.

[15] Mariangela Lisanti, Siddharth Mishra-Sharma, Lina Necib, and Benjamin R. Safdi. Deciphering Contributions to the Extragalactic Gamma-Ray Background from $2 \mathrm{GeV}$ to $2 \mathrm{TeV}$. Astrophys. J., 832(2):117, 2016.

[16] Cecilia Lunardini, Gregory S. Vance, Kimberly L. Emig, and Rogier A. Windhorst. Are starburst galaxies a common source of high energy neutrinos and cosmic rays? JCAP, 10:073, 2019.

[17] Kohta Murase, Shigeo S. Kimura, and Peter Meszaros. Hidden Cores of Active Galactic Nuclei as the Origin of Medium-Energy Neutrinos: Critical Tests with the MeV Gamma-Ray Connection. Phys. Rev. Lett., 125(1):011101, 2020.

[18] Timothy A. D. Paglione, Alan P. Marscher, James M. Jackson, and David L. Bertsch. Diffuse Gamma-Ray Emission from the Starburst Galaxy NGC 253. Astrophys. J., 460:295, March 1996.

[19] Andrea Palladino, Anatoli Fedynitch, Rasmus W. Rasmussen, and Andrew M. Taylor. IceCube Neutrinos from Hadronically Powered Gamma-Ray Galaxies. JCAP, 09:004, 2019.

[20] Andrea Palladino, Xavier Rodrigues, Shan Gao, and Walter Winter. Interpretation of the diffuse astrophysical neutrino flux in terms of the blazar sequence. Astrophys. J., 871(1):41, 2019.

[21] Enrico Peretti, Pasquale Blasi, Felix Aharonian, and Giovanni Morlino. Cosmic ray transport and radiative processes in nuclei of starburst galaxies. Mon. Not. Roy. Astron. Soc., 487(1):168180, 2019.

[22] Enrico Peretti, Pasquale Blasi, Felix Aharonian, Giovanni Morlino, and Pierre Cristofari. Contribution of starburst nuclei to the diffuse gamma-ray and neutrino flux. Mon. Not. Roy. Astron. Soc., 493(4), 2020.

[23] Cesar Rojas-Bravo and Miguel Araya. Search for gamma-ray emission from star-forming galaxies with Fermi LAT. Mon. Not. Roy. Astron. Soc., 463(1):1068-1073, 2016.

[24] Todd A. Thompson, Eliot Quataert, Eli Waxman, Norman Murray, and Crystal L. Martin. Magnetic fields in starburst galaxies and the origin of the fir-radio correlation. Astrophys. J., 645:186-198, 2006. 